\section{SAT0401 SWOLLEN JOINTS ARE ASSOCIATED WITH ULTRASOUND POWER DOPPLER SYNOVITIS, WHEREAS TENDER JOINTS INTHE ABSENCE OF SWELLING ARE NOT: AN ANALYSIS OF AGREEMENT AND CORRELATION IN VERY EARLY DMARD NAÏVE PSORIATIC ARTHRITIS}

S. Dubash ${ }^{1}$, O. Alabas ${ }^{1}$, X. Michelena ${ }^{1}$, L. Garcia-Montoya ${ }^{1}$, G. De Marco', M. Merashli ${ }^{1}$, R. Wakefield ${ }^{1}$, P. Helliwell ${ }^{1}$, D. Mcgonagle ${ }^{1}$, A. L. Tan ${ }^{1}$, P. Emery ${ }^{1}$, H. Marzo-Ortega ${ }^{1} .{ }^{1}$ NIHR LBRC, Leeds Teaching Hospitals Trust \& LIRMM, University of Leeds, Leeds, Leeds, United Kingdom

Background: Ultrasound (US) is an imaging adjunct to clinical joint examination adding sensitivity and objectivity to the assessment of inflammation. Previous studies in PSA have shown disparity between ultrasound and clinical findings with significant subclinical joint inflammation. A clinical challenge in PsA is to interpret tender joints (TJ) that are not swollen (SJ). As US is not widely used alignment of clinical with US assessment is needed to determine its future role. Objectives: To determine how joint clinical examination relates to US findings in very early DMARD naïve PsA.

Methods: Newly diagnosed DMARD naïve PsA patients, fulfilling CASPAR criteria, were recruited into the Leeds Spondyloarthropathy Register for Research and Observation (SpARRO), a prospective observational cohort study. US examination of 48 joints per patient was conducted by trained ultra-sonographers, blinded to clinical details with semi-quantitative scoring (0-3) for gray scale (GS) and power Doppler (PD). TJ and SJ counts were independently recorded. Cross-sectional baseline analysis was performed. The prevalence-adjusted and bias-adjusted kappa (PABAK) was calculated to determine agreements between clinical and US parameters. Spearman's rank correlation coefficient was calculated to identify permutations of $T J / S J$ correlating with $G S \geq 2, P D \geq 1$ or both.

Results: A total 5927 joints were scanned in 155 PsA patients. The mean age was 44.4 years, (SD 12.8), median disease duration 5.1 weeks (0.4-13.1); median $\mathrm{TJC}=7$ (3-14) and SJC=2 (1-7). Oligoarthritis was present in 63.9\% (99/155). US GS $\geq 2$ was frequently detected in the feet at MTPs1-4 (37.4- 53.6\%) and wrists (26.5- 33.6\%). PD was most prevalent at wrists (17.5\%) and MTP1 (12.6\%) but observed less in other joints. Erosions were less frequent, the commonest site being MTP5 (17/310, 5.4\%).

Overall, SJ demonstrated high agreement $(p<0.001)$ with US synovitis (GS $\geq 2$ and/or $P D \geq 1$ ). High agreement was equivalent between combined $G S \geq 2$ and $P D \geq 1$ compared with $P D \geq 1$ alone $(p<0.001)$ indicating it was predominantly driven by PD. Agreement with TJ and US was consistently lower yet still significant $(p<0.001)$. Combinations of TJ/SJ were explored with US synovitis (table 1 ). Correlation was significant for $\mathrm{T}+\mathrm{S}+$ and $\mathrm{PD} \geq 1$ at wrists, MCP1-5, PIP2-5, MCP3-4 $(p<0.001)$; DIP2 $(p<0.05)$, knees and ankles $(p<0.01)$ but weaker correlation in MTP3,4. In contrast, poor correlation was observed in the T+ S- group for most joints.

Table 1. Agreement between TJ or SJ with $G S \geq 2 \& P D \geq 1$ and correlations for tender with/ without swollen combinations for right sided hand/feet joints.

\begin{tabular}{|c|c|c|c|c|c|c|}
\hline \multirow[b]{2}{*}{ Joint (Right) } & \multicolumn{2}{|c|}{ Tender } & \multicolumn{2}{|c|}{ Swollen } & \multirow{2}{*}{$\frac{T+S-}{r}$} & \multirow{2}{*}{$\frac{T+S+}{r}$} \\
\hline & $\mathrm{A}(\%)$ & PABAK & A (\%) & PABAK & & \\
\hline Wrist & 75.5 & $0.51^{\star}$ & 89.1 & $0.78^{\star}$ & -0.09 & $0.35^{*}$ \\
\hline MCP1 & 84.1 & $0.68^{*}$ & 87.5 & $0.75^{*}$ & 0.09 & $0.44^{*}$ \\
\hline MCP2 & 77.7 & $0.55^{*}$ & 83.1 & $0.66^{*}$ & 0.08 & $0.35^{*}$ \\
\hline MCP3 & 79.1 & $0.58^{\star}$ & 84.5 & $0.69^{\star}$ & 0.005 & $0.50^{*}$ \\
\hline MCP4 & 78.4 & $0.57^{*}$ & 86.4 & $0.72^{*}$ & 0.07 & $0.22 \dagger$ \\
\hline MCP5 & 87.8 & $0.76^{*}$ & 95.6 & $0.91^{*}$ & -0.03 & $0.49^{\star}$ \\
\hline MTP1 & 69.8 & $0.40^{*}$ & 83.9 & $0.68^{*}$ & -0.03 & \\
\hline MTP2 & 79.1 & $0.58^{\star}$ & 90.5 & $0.81^{*}$ & 0.06 & 0.11 \\
\hline MTP3 & 77.0 & $0.54^{\star}$ & 88.5 & $0.77^{*}$ & 0.05 & $0.22 \ddagger$ \\
\hline MTP4 & 77.7 & $0.55^{\star}$ & 87.2 & $0.74^{\star}$ & -0.002 & $0.23 \ddagger$ \\
\hline MTP5 & 79.9 & $0.60^{*}$ & 89.9 & $0.80^{*}$ & 0.15 & 0.09 \\
\hline
\end{tabular}

$T_{+}=$tender, $S_{+}=$swollen, $S_{-}=$not swollen, $A=$ agreement $(\%), r=$ coefficient, $\dagger p<0.05, \ddagger$ $\mathrm{p}<0.01,{ }^{*} \mathrm{p}<0.001$

Conclusion: Swollen joints demonstrate higher agreement with US synovitis ( $P D \geq 1$ alone or $G S \geq 2 \& P D \geq 1$ combined) than tender joints in early PsA. In addition, joints that are tender but not swollen have poor correlation with US synovitis at the individual joint level indicating that swelling is a better clinical discriminator of active synovitis, and factors other than synovial inflammation may drive tenderness in very early, DMARD naïve PsA. These results suggest re-appraisal of clinical joint counts is needed to refine treatment decision making in early PsA. Disclosure of Interests: Sayam Dubash: None declared, Oras Alabas: None declared, Xabier Michelena: None declared, Leticia Garcia-Montoya: None declared, Gabriele De Marco: None declared, Mira Merashli: None declared, Richard Wakefield Speakers bureau: Novartis, Janssen, GE, Philip Helliwell: None declared, Dennis McGonagle Grant/research support from: Janssen
Research \& Development, LLC, Ai Lyn Tan: None declared, Paul Emery Grant/ research support from: AbbVie, Bristol-Myers Squibb, Merck Sharp \& Dohme, Pfizer, Roche (all paid to employer), Consultant of: AbbVie (consultant, clinical trials, advisor), Bristol-Myers Squibb (consultant, clinical trials, advisor), Lilly (clinical trials, advisor), Merck Sharp \& Dohme (consultant, clinical trials, advisor), Novartis (consultant, clinical trials, advisor), Pfizer (consultant, clinical trials, advisor), Roche (consultant, clinical trials, advisor), Samsung (clinica trials, advisor), Sandoz (clinical trials, advisor), UCB (consultant, clinical trials, advisor), Helena Marzo-Ortega Grant/research support from: Janssen, Novartis, Consultant of: Abbvie, Celgene, Eli Lilly, Janssen, Novartis, Pfizer, UCB, Speakers bureau: Abbvie, Celgene, Eli Lilly, Janssen, Novartis, Pfizer, Takeda, UCB DOI: 10.1136/annrheumdis-2020-eular.781

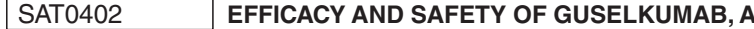 MONOCLONAL ANTIBODY SPECIFIC TO THE P19- SUBUNIT OF INTERLEUKIN-23, THROUGH WEEK 52 OF A PHASE 3, RANDOMIZED, DOUBLE-BLIND, PLACEBO-CONTROLLED STUDY CONDUCTED IN BIOLOGIC-NAÏVE PATIENTS WITH ACTIVE PSORIATIC ARTHRITIS}

I. Mcinnes ${ }^{1}$, P. Rahman ${ }^{2}$, A. B. Gottlieb ${ }^{3}$, E. C. Hsia ${ }^{4,5}$, A. Kollmeier ${ }^{4}$, X. L. Xu ${ }^{4}$, R. A. Subramanian ${ }^{4}$, P. Agarwal ${ }^{4}$, S. Sheng ${ }^{4}$, Y. Jiang ${ }^{4}$, B. Zhou ${ }^{4}$, D. Van der Heijde $^{6}$, P. J. Mease ${ }^{7} .{ }^{1}$ Univ Glasgow, Glasgow, United Kingdom; ${ }^{2}$ Memorial Univ Newfoundland, St John's, Canada; ${ }^{3}$ Icahn School of Med Mt Sinai, New York, United States of America; ${ }^{4}$ Janssen Research \& Development, LLC, Spring House, United States of America; ${ }^{5} U$ Penn Med Ctr, Philadelphia, United States of America; ${ }^{6}$ Leiden Univ Medical Ctr, Leiden, Netherlands; ${ }^{7}$ Swedish Med Ctr/Providence St Joseph Health and U Wash School of Med, Seattle, United States of America

Background: Guselkumab (GUS), a monoclonal antibody that specifically binds to the p19-subunit of IL-23, is approved to treat psoriasis. Through Week24 (W24) of the $\mathrm{Ph} 3$, double-blind, placebo (PBO)-controlled trial in biologic-naïve pts with active PsA (DISCOVER-2), GUS every 4 or 8 weeks (Q4W or Q8W) demonstrated efficacy for joint \& skin symptoms and inhibition of structural damage progression (Q4W), and was well tolerated.

Objectives: Assess GUS efficacy and safety through W52.

Methods: Biologic-naïve adults with active PsA ( $\geq 5$ swollen $+\geq 5$ tender joints; CRP $\geq 0.6 \mathrm{mg} / \mathrm{dL}$ ) were randomized (1:1:1) to GUS $100 \mathrm{mg}$ Q4W; GUS $100 \mathrm{mg}$ at W0, W4, Q8W; or PBO. At W24, PBO pts crossed over to GUS $100 \mathrm{mg}$ Q4W (PBO X Q4W). ACR response rates at W52, based on nonresponder imputation (NRI) for missing data and as observed in pts who continued study agent at W24, are shown. Observed data for additional endpoints, including PsA-modified van der Heijde Sharp (vdH-S) scores derived from blinded radiographic images collected at W0, W24, W52 (or at d/c) and scored in a new Read Campaign, are shown.

Results: $712 / 739(96.3 \%)$ randomized \& treated pts continued study agent at W24; 689/739 (93.2\%) completed Wk52. NRI ACR20 response rates continued to increase after W24, and at W52 were $70.6 \%$ for GUS Q4W and $74.6 \%$ for GUS Q8W (Fig 1A). Similar response patterns were observed for the more stringent ACR50/70 criteria (Fig 1C,E). Observed ACR (Fig, 1B,D,F), IGA, PASI \& MDA VLDA responses; dactylitis \& enthesitis resolution; and mean improvements in HAQ-DI and SF-36 PCS/MCS scores were also sustained through W52 in pts receiving Q4W \& Q8W; W52 data for PBO X Q4W pts were generally consistent with other GUS-treated pts (Fig 1, Table 1). Changes in vdH-S scores were

Table 1. Observed Efficacy ${ }^{1}$

\begin{tabular}{lcccccc}
\hline & GUS & Q4W & GUS & Q8W & $\begin{array}{r}\text { PBO X } \\
\text { (W0-24) }\end{array}$ & $\begin{array}{c}\text { GUS Q4W } \\
\text { (W24-52) }\end{array}$ \\
\hline Data are \% unless otherwise stated & W24 & W52 & W24 & W52 & W24 & W52 \\
Dactylitis at W0, n & 116 & 111 & 107 & 105 & 95 & 93 \\
Resolution & 68.1 & 81.1 & 60.7 & 81.9 & 41.1 & 78.5 \\
Enthesitis at W0, n & 165 & 160 & 151 & 148 & 172 & 168 \\
Resolution & 45.5 & 60.0 & 57.6 & 65.5 & 32.6 & 67.3 \\
$\geq 3 \%$ BSA psoriasis, IGA $\geq 2$ at W0, $\mathrm{n}$ & 176 & 173 & 172 & 170 & 176 & 172 \\
IGA 0/1 + $\geq 2$-grade decrease & 71.0 & 84.4 & 72.1 & 77.1 & 19.9 & 84.3 \\
PASI75 & 81.8 & 91.9 & 80.8 & 88.8 & 23.3 & 88.4 \\
PASI90 & 63.6 & 81.5 & 70.3 & 77.1 & 10.2 & 76.7 \\
PASI100 & 46.6 & 61.3 & 46.5 & 54.7 & 2.8 & 55.2 \\
HAQ-DI, $n$ & 234 & 229 & 238 & 234 & 237 & 230 \\
Mean change & -0.4 & -0.5 & -0.4 & -0.5 & -0.2 & -0.4 \\
SF-36 scores, $n$ (mean change) & 234 & 229 & 238 & 234 & 237 & 230 \\
Physical Component - PCS & 7.2 & 9.0 & 7.8 & 9.5 & 3.8 & 8.1 \\
Mental Component - MCS & 4.1 & 4.1 & 4.5 & 4.5 & 2.2 & 4.3 \\
MDA/VLDA, n & 234 & 228 & 238 & 234 & 238 & 231 \\
MDA & 19.7 & 36.8 & 26.5 & 32.9 & 6.3 & 31.6 \\
VLDA & 5.1 & $12.2^{2}$ & $4.6^{3}$ & 17.1 & 1.3 & 6.9 \\
\hline
\end{tabular}

${ }^{1}$ Randomized pts still on study agent at $\mathrm{W} 24 ;{ }^{2} \mathrm{~N}=229 ;{ }^{3} \mathrm{~N}=237$ 